\title{
TrkB/BDNF-Dependent Striatal Plasticity and Behavior in a Genetic Model of Epilepsy: Modulation by Valproic Acid
}

\author{
Veronica Ghiglieri ${ }^{1,5}$, Carmelo Sgobio', ${ }^{1,5}$, Stefano Patassini ${ }^{2}$, Vincenza Bagetta', Anna Fejtova ${ }^{3}$, \\ Carmela Giampà ${ }^{2}$, Silvia Marinucci', Alexandra Heyden ${ }^{3}$, Eckart D Gundelfinger ${ }^{3}$, Francesca R Fusco ${ }^{2}$, \\ Paolo Calabresi ${ }^{1,4}$ and Barbara Picconi*,I \\ 'Laboratorio di Neurofisiologia, Fondazione Santa Lucia, IRCCS, via del Fosso di Fiorano 64, Rome, Italy; 'Laboratorio di Neuroanatomia, \\ Fondazione Santa Lucia, IRCCS, Rome, Italy; ${ }^{3}$ Leibniz Institute for Neurobiology, Brenneckestr 6, Magdeburg, Germany; ${ }^{4}$ Clinica Neurologica, \\ Dip. Specialità Medico Chirurgiche e Sanità Pubblica, Università di Perugia, S. Maria della Misericordia, Perugia, Italy
}

In mice lacking the central domain of the presynaptic scaffold Bassoon the occurrence of repeated cortical seizures induces cell-typespecific plasticity changes resulting in a general enhancement of the feedforward inhibition within the striatal microcircuit. Early antiepileptic treatment with valproic acid (VPA) reduces epileptic attacks, inhibits the emergence of pathological form of plasticity in fast-spiking (FS) interneurons and restores physiological striatal synaptic plasticity in medium spiny (MS) neurons. Brain-derived neurotrophic factor (BDNF) is a key factor for the induction and maintenance of synaptic plasticity and it is also implicated in the mechanisms underlying epilepsy-induced adaptive changes. In this study, we explore the possibility that the TrkB/BDNF system is involved in the striatal modifications associated with the Bassoon gene (Bsn) mutation. In epileptic mice abnormal striatum-dependent learning was paralleled by higher TrkB levels and an altered distribution of BDNF. Accordingly, subchronic intrastriatal administration of k252a, an inhibitor of TrkB receptor tyrosine kinase activity, reversed behavioral alterations in Bsn mutant mice. In addition, in vitro manipulations of the TrkB/BDNF complex by k252a, prevented the emergence of pathological plasticity in FS interneurons. Chronic treatment with VPA, by reducing seizures, was able to rebalance TrkB to control levels favoring a physiological redistribution of BDNF between MS neurons and FS interneurons with a concomitant recovery of striatal plasticity. Our results provide the first indication that BDNF is involved in determining the striatal alterations occurring in the early-onset epileptic syndrome associated with the absence of presynaptic protein Bassoon.

Neuropsychopharmacology (2010) 35, I53I-1540; doi:I0.1038/npp.2010.23; published online 3 March 2010

Keywords: Bassoon mutant; procedural learning; electrophysiology; dorsolateral striatum; parvalbumin-positive interneurons; medium spiny neurons

\section{INTRODUCTION}

In the striatum of mice lacking the central domain of Bassoon gene (Bsn) corticostriatal synaptic plasticity changes in response to early-onset epilepsy (Ghiglieri et al, 2009). In epileptic mice, striatal medium spiny (MS) neurons show morphological alterations and a reduced long-term potentiation (LTP) while fast-spiking (FS) parvalbumin (PV)-positive GABAergic interneurons are more numerous and express a short-term potentiation that is not observed in the wild-type mice. Interestingly, early therapeutic intervention with valproic acid (VPA), a broad

*Correspondence: Dr B Picconi, Laboratory of Neurophysiology, Fondazione Santa Lucia, via del Fosso di Fiorano 64, Rome 00143 , Italy, Tel: + 3906 501703152, Fax: + 3906 501703316,

E-mail: b.picconi@hsantalucia.it

${ }^{5}$ These two authors contributed equally to this paper.

Received 27 October 2009; revised 22 January 2010; accepted 4

February 2010 spectrum antiepileptic drug (AED), is able to prevent the cell-type-specific functional alterations induced by chronic seizures. In the healthy brain, structural and functional changes represent the basis of plasticity and are strongly regulated by brain-derived neurotrophic factor (BDNF) (Lu, 2003; Poo, 2001). Corticostriatal neurons deliver BDNF through activity-dependent release into the striatum in which it binds to its high-affinity receptor, the tyrosin kinase tropomyosin-related kinase B (TrkB) receptor, leading to the activation of distinct intracellular pathways that control neurite growth, synaptic plasticity, proliferation, and survival (Zuccato and Cattaneo, 2009). It is noteworthy that BDNF is specifically required for the maturation of inhibitory GABAergic synapses (Bartrup et al, 1997; Marty et al, 1996; Yamada et al, 2002) and the regulation of interneuronal properties (Berghuis et al, 2004). BDNF also differently modulates development and function of excitatory and inhibitory synaptic transmission (Bolton et al, 2000). 
Besides the control of these constitutive aspects, BDNF has a pivotal role in the mechanisms underlying epilepsyinduced adaptive changes.

In fact, it has been reported that in experimental models and in epileptic patients both BDNF mRNA and protein levels are increased in the foci of seizures (Gall, 1993; Humpel et al, 1993; Isackson et al, 1991; Schmidt-Kastner et al, 1996; Takahashi et al, 1999; Jankowsky and Patterson, 2001). It has also been shown that striatal mRNA of TrkB is transiently elevated after seizures (Merlio et al, 1993; Salin et al, 1995) and that epileptiform activity induces a strong upregulation of cortical TrkB (Wyneken et al, 2001, 2003). All these data support the idea that TrkB/BDNF signaling pathway is a key factor in the development and control of seizures, although the question whether BDNF has a permissive or protective function in the epileptogenesis is still debated. Moreover, in spite of the potential relevance of these actions for synaptic plasticity and striatal-dependent learning, the possible role of BDNF in the corticostriatal plasticity during epilepsy has not been considered yet. To analyze whether TrkB/BDNF system is specifically involved in the striatal modifications associated with Bsn mutation, we first analyzed behavioral and plastic changes in Bsn mutants after in vivo inhibition of TrkB receptor activity. Moreover, we performed quantification of striatal TrkB levels on immunoblots and immunohistochemical analysis of BDNF protein expression pattern in striatal MS neurons and FS interneurons in both wild-type and epileptic mice. Then, to explore whether these modifications may depend on chronic seizure activity, we measured TrkB levels and evaluated BDNF distribution in the striatum of epileptic mice after chronic VPA treatment.

\section{MATERIALS AND METHODS}

\section{Animals}

Mice lacking part of the Bassoon gene (Bsn $\Delta \mathrm{Ex} 4 / 5)$ were generated as described earlier (Altrock et al, 2003), backcrossed into C57/bl6 mouse strain, and then crossed with SV129 strain yielding mice with a mixed C57/bl6-SV129 genetic background for mutants and wild types. Genotyping was performed by polymerase chain reaction as described earlier (Altrock et al, 2003); age-matched wild-type littermates were used as controls. At the beginning of the experiment, animals were 2-3 months old and their weights ranged from 23 to $28 \mathrm{~g}$. They were housed in a temperaturecontrolled room $\left(22^{\circ} \mathrm{C}\right)$ with a light-dark $12: 12$ cycle (lights on $0700-1900 \mathrm{~h}$ ). Food and water were given ad libitum. All experiments were carried out according to the guidelines on the ethical use of animals from the European Communities Council Directive of 24 November 1986 (86/609/EEC) and from the National Ministry of Health (DM 222/2008).

\section{Surgery Procedures}

Adult Bsn mutants and wild-type controls weighing $28-30 \mathrm{~g}$ were anesthetized by i.p. injection of chloral hydrate (400 mg/kg) (Merk KGaA, Darmstadt, Germany) and placed into a stereotaxic frame (Stoelting, Co, USA). A guide cannula (inner diameter $0.25 \mu \mathrm{m}$, outer diameter $0.50 \mu \mathrm{m}$ )
(Unimed, Lausanne, Switzerland) was placed into the dorsal striatum according to Paxinos and Franklin (2007) at + $1.1 \mathrm{~mm}$ from bregma, $2.0 \mathrm{~mm}$ lateral to the midline, and $2.5 \mathrm{~mm}$ from dura mater. The cannula was permanently secured to the skull using cranioplastic cement (Glasionomer Cement CX Plus, Shofu, Kyoto, Japan) and closed with a dummy cannula (Unimed). After the surgery, mice were allowed to recover for 3 days before drug administration. Only animals showing normal neurological functions and no evidence of infection were randomized into experiments. Brain sections were examined under a light microscope to allow visual checking of cannula placement and mice without proper positioning of cannula were excluded from analysis.

\section{Behavioral Measurements}

Eight mice per group were used in the training experiments. Active avoidance task requires to learn that an explicit conditional stimulus (CS: light) predicts the delivery of a negative unconditioned stimulus (US: footshock). The test was carried out in a battery of six two-way shuttle boxes $(40 \times 10 \times 15 \mathrm{~cm})$. Each box was divided into two compartments by a partition with an opening at the floor level. The boxes had a transparent cover with a light bulb $(10 \mathrm{~W})$ attached above each compartment. The floor was made of a stainless steel grid. Mice were subjected to one active avoidance session (duration $30 \mathrm{~min}, 60$ avoidance trials) for 5 consecutive days. Each trial consisted of a $30 \mathrm{~s}$ light signal (CS) presented in one compartment $5 \mathrm{~s}$ before the onset of an electric foot shock (7 mA, $25 \mathrm{~s})$ in the same compartment (US). Two dependent variables were recorded: escape responses (crossings during US presentation), and conditioned responses (crossings occurring within $5 \mathrm{~s}$ of $\mathrm{CS}$ ). Foot-shock sensitivity was evaluated by placing mice in a Plexiglas cage $(28 \mathrm{~cm}$ long $\times 28 \mathrm{~cm}$ wide $\times 10 \mathrm{~cm}$ high $)$ with a grid floor connected to a shock producer. Mice were individually placed in the cage and their pain thresholds were evaluated by increasing current intensity from 0 to a maximum of $0.6 \mathrm{~mA}$. The minimal intensity eliciting vocalization and jumping was retained as the score. Mice failing to squeak were given the maximum score of $0.6 \mathrm{~mA}$. Two-way ANOVA was performed to compare means with one factor between groups (genotype) and one factor within groups (day). Mutants showing episodic thigmotaxis and stereotypies were not included in the statistical analysis to avoid bias in scoring motor behaviors.

\section{Electrophysiological Experiments}

Eight adult mice (2-3 months old, weighting 23-28 g) were used per groups for all the electrophysiological experiments. Animals were killed by cervical dislocation to obtain coronal corticostriatal slices for electrophysiological recordings (Calabresi et al, 1992). The slices $(200-300 \mu \mathrm{m})$ were prepared from tissue blocks of the brain with the use of a vibratome. A single slice was transferred to a recording chamber and submerged in a continuously flowing Krebs' solution $\left(33^{\circ} \mathrm{C}, 2-3 \mathrm{ml} / \mathrm{min}\right)$ gassed with $95 \% \mathrm{O}_{2}$ and $5 \%$ $\mathrm{CO}_{2}$. The composition of the control solution was (in $\mathrm{mM}$ ): $126 \mathrm{NaCl}, 2.5 \mathrm{KCl}, 1.2 \mathrm{MgCl} 2,1.2 \mathrm{NaH} 2 \mathrm{PO} 4,2.4 \mathrm{CaCl} 2,11$ glucose, and $25 \mathrm{NaHCO} 3$. In all the experiments, the 
intracellular recording electrodes were filled with $2 \mathrm{M} \mathrm{KCl}$ (30-60 M $\Omega$ ). Signals were recorded using an Axoclamp 2B amplifier (Molecular Devices, Sunnyvale, CA, USA), showed on a separate oscilloscope, stored, and analyzed on a digital system (pClamp 9, Axon Instruments). For synaptic stimulation, bipolar electrodes were located in the white matter between the cortex and the dorsolateral striatum to activate corticostriatal fibers. For LTP protocol, at the beginning of intracellular recordings, magnesium ions were omitted from the medium to increase the NMDA-mediated component of the excitatory postsynaptic potential (EPSP). As conditioning tetanus (high-frequency stimulation, HFS), we used three trains ( $3 \mathrm{~s}$ duration, $100 \mathrm{~Hz}$ frequency, at 20 -s intervals). During tetanic stimulation, the intensity was increased to suprathreshold levels. Quantitative data on EPSP modifications induced by tetanic stimulation are expressed as a percentage of the controls, the latter representing the mean of responses recorded during a stable period (15-20 $\mathrm{min}$ ) before the tetanus. Values given in the text and in the figures are mean \pm SEM of changes in the respective cell populations. Two-way ANOVA, followed by Bonferroni post hoc test, and Student's $t$-test were used to compare the means between genotypes and pre- $v s$ posttetanic, respectively.

\section{Immunohistochemistry and Quantitative Analysis}

For the histological analysis three animals for each group were used. Forty-micrometer-thick coronal sections were cut on a microtome (Microm, Walldorf, Germany) and collected in sequence in 24-well plates containing $1 \mathrm{ml}$ of PB. Striatal sections were processed free-floating for parvalbumin (PV) and Calbindin (Calb) immunofluorescence, incubated with a rabbit anti-parvalbumin $(1: 500$; Chemicon, CA, USA) and a mouse anti-Calb antibody (1:500; Sigma Aldrich, Mi, Italy) and subsequently with a secondary Cy2-conjugated antibody donkey anti-mouse IgG and Cy3- conjugated donkey anti-rabbit IgG $(1: 100$; Jackson Immunoresearch Laboratories, West Grove, PA, USA). Specificity of the antibodies was tested by primary omission controls, blocking peptide immunoreaction, and dilution series. Sections were then mounted in gelatin-coated slides, air-dried, and coverslipped with GEL/MOUNT (Biomeda, Foster City, CA, USA). All the sections were batch-processed at the same time. For each section, three equally spaced photomicrographs of the dorsolateral part of the striatum were taken using the $40 \times$ magnification of a confocal microscope CLSM (Zeiss LSM 510). For each mouse, PV and Calb-positive cells were counted at four different rostrocaudal levels of the striatum. The coordinates according to Paxinos and Franklin (2007) were $+1.10,+0.62,+0.14$, and $-0.34 \mathrm{~mm}$ from bregma. For each brain, we examined three confocal images of dorsolateral striatum, using as landmark the corpus callosum. A total of 24 confocal images for each one animal was examined. A reconstructed striatal area in Supplementary Figure S1 describes the three subfields into which the striatum was subdivided that were used for the cell counts.

The number of cells was evaluated in each picture acquired using Image J software (http://rsb.info.nih.gov/ij). The ratio of the total amount of cells for each labeling was calculated following the formula: PV-positive cells/
Calb-positive cells; and compared with a one-way ANOVA. Furthermore, to analyze the abundance of BDNF protein in PV-positive interneurons, the sections were double labeled with an antibody against BDNF (mouse anti-BDNF, $1: 200$, Immunological Sciences, Italy) and rabbit anti-PV (1:500, Chemicon). Sections were examined under an epi-illumination fluorescent microscope (Zeiss Axioskop 2) and a confocal Laser scanning microscope (CLSM) Zeiss LSM510. Digital images were acquired using the Zeiss LSM510 computer program. The number of PV-interneurons that were labeled for BDNF, and devoid of it, was counted. The density of PV-positive cells that were BDNF immunoreactive was thus obtained. The levels of colocalization in each field were averaged across all fields and a statistical analysis by means of one-way ANOVA was used setting $p<0.05$.

\section{Western Blotting Analysis}

Six wild-type and six mutant mice with ages of 2-3 months were killed by cervical dislocation. The striatum was prepared from the brains, immediately frozen in liquid nitrogen and stored at $-80^{\circ} \mathrm{C}$. For analysis of protein levels in total homogenates, tissue from individual mice was homogenized in buffer $\mathrm{H}$ ( $5 \mathrm{mM}$ TrisHcl, $0,5 \%$ TritonX-100 with Complete protease inhibitors (Roche)) using Teflon homogenizer (900 r.p.m., 12 strokes). To isolate PSDenriched protein fraction the initial homogenate was kept for $1 \mathrm{~h}$ at $4{ }^{\circ} \mathrm{C}$ to extract TritonX-100 soluble proteins and centrifuged at 100000 r.p.m. at $4{ }^{\circ} \mathrm{C}$ for $1 \mathrm{~h}$. To increase the purity of isolated PSD-enriched material the pellet was washed by rehomogenization in buffer $\mathrm{H}$ followed by centrifugation. The supernatants of both centrifugation steps were pooled. Proteins of both resulting fractions were precipitated with acetone. SDS-PAGE and western blotting were performed according to standard procedures. Antibody against alpha-tubulin (DM1A, Sigma) and TrkB (H-181) antibody (Santa Cruz) and secondary antibody coupled to Odyssey IRDye 680 or Odyssey IRDye 800 were used. The signals were scanned and quantified on Odyssey imaging system (LI-COR). The levels of TrkB in each probe were normalized to alpha-tubulin signal.

\section{Drugs}

For in vivo administration k252a (Alomone Labs, Jerusalem, Israel) was dissolved in dimethylsulfoxide and diluted to $9.34 \mathrm{ng} / \mu \mathrm{l} .(20 \mu \mathrm{M})$ in Krebs'solution. Intrastriatal infusion of either k252a or vehicle were given to hand-restrained conscious animals $20 \mathrm{~min}$ previous each session of active avoidance testing. Cannulas used for administration were attached by polyethylene tubing to a $10 \mu \mathrm{l}$ Hamilton syringe (Sigma-Aldrich, Milano, Italy) which, in turn, was driven by a microinfusion pump (World Precision Instruments, Sarasota, FL, USA) at a rate of $0.2 \mu \mathrm{l} / \mathrm{min}$ (total volume of $1 \mu \mathrm{l}$ ). Injection cannulas were left in place for $2 \mathrm{~min}$ after the infusion was completed.

For in vitro electrophysiological recordings $\mathrm{k} 252 \mathrm{a}$ was applied by diluting it in Krebs'solution to the final concentration of $200 \mathrm{nM}$ and by switching the perfusion from control to drug-containing solution. Sodium Valproate (Depakin, $200 \mathrm{mg} / \mathrm{ml}$; Sanofi Aventis, Mi, Italy) 
was added into drinking water at a concentration of $100 \mathrm{mg} / 100 \mathrm{ml}$ to administer a daily dose of approximately $400 \mathrm{mg} / \mathrm{kg}$.

\section{RESULTS}

\section{Increased Active Avoidance Performances in Bsn Mutant Mice}

Striatal-dependent learning abilities of $B s n$ mutant and wild-type mice were assessed in the two-way active avoidance test. The test is based on the capability to form a simple CS-US association and extensive findings indicate that the formation of such associations largely depend on the dorsolateral striatum (White and McDonald, 2002). In the present experiment, eight mice for each genotype were subjected to daily sessions consisting of 30 CS-US presentations, for 5 consecutive days. On each session, the number of conditioned responses and inter-trial crossings was scored. Our results show that performances increased in each group as training proceeded (significant effect of session: $\mathrm{F}(4,56)=11.56 ; p<0.001)$. Interestingly, $B s n \mathrm{mu}-$ tant mice showed higher active avoidance scores than wildtype mice (significant effect of genotype: $\mathrm{F}(1,14)=12.60$; $p<0.01$ ) (Figure 1a, left panel) while the number of inter-trial crossings, one main index of sustained motility, was similar in both genotypes (Figure 1a, right panel).

\section{Inhibition of TrkB Tyrosine Kinase Activity Reduces Procedural Learning of Bsn Mutant Mice to Control Levels}

To analyze the role of TrkB/BDNF system in the striataldependent procedural learning, wild-type mice and $B s n$ mutants receiving intrastriatal administration of the TrkB inhibitor k252a were subjected to a battery of active avoidance testing. Fifteen minutes after the injection of drug or vehicle, animals were subjected to a 30 -min session, once a day, for 5 days. Also in these groups the performances increased as training proceeded (significant effect of session: $\mathrm{F}(4,28)=12.80 ; p<0.001$ ) (Figure $1 \mathrm{~b}$, left panel) and the number of inter-trial crossings was similar (Figure $1 \mathrm{~b}$, right panel). However, after administration of k252a $(20 \mu \mathrm{M}$, intrastriatum), task performances were worsened in wild-type mice (data not shown) while scores of treated Bsn mutant mice were similar to vehicle-injected wild-type controls (Figure 1b, left panel).
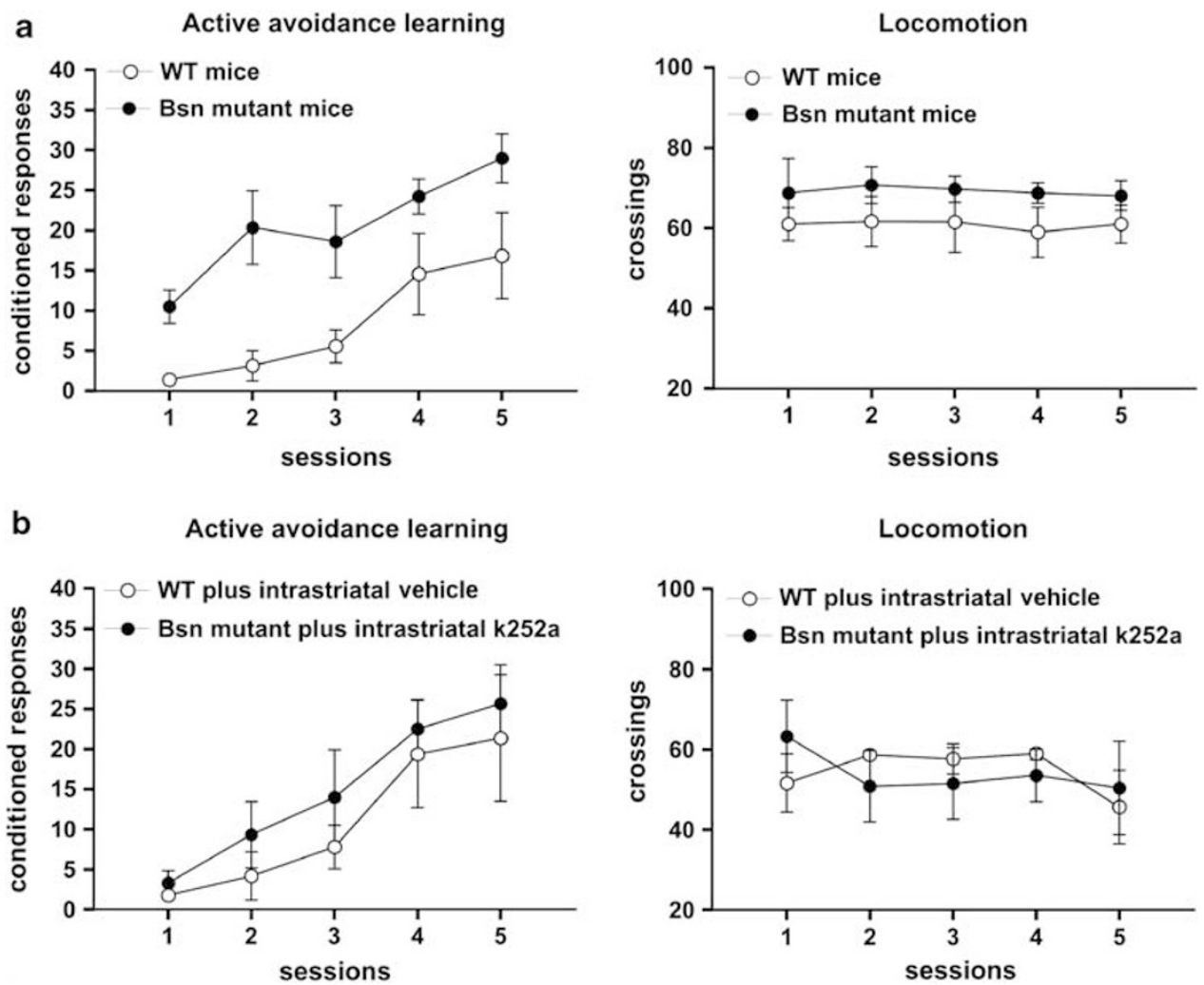

Figure I TrkB dependent increased procedural learning in Bsn mutant mice. Corticostriatal-dependent learning capabilities of Bsn mutants and wild-type littermates were tested using a two-way active avoidance test. (a) Graph on the left shows the mean number of avoidance responses per day \pm SEM recorded for each group. Two-way ANOVA revealed an effect of genotype $(F(I, I 4)=12.60 ; p<0.01$ ), indicating that performances were higher in Bsn mutants than in wild-type mice. Mean number of crossings between the two compartments per day \pm SEM are shown on the right panel. Two-way ANOVA revealed no significant group main effect, indicating that locomotion did not significantly differ between genotypes. (b) Subchronic intrastriatal administration of k252a $(20 \mu \mathrm{M})$, an inhibitor of TrkB tyrosine kinase activity, in epileptic mice, caused a reduction of task performances to control values, as shown by the graph on the left. Statistical comparisons between the two genotypes show that subchronic k252a did not affect the number of crossing, which was similar in the two experimental groups (right panel). 
In Vitro TrkB Antagonism Abolishes Short-Term Plasticity in FS Interneurons of Bsn Mutant Mice

We have previously observed that, after HFS, FS interneurons recorded from $B s n$ mutant mice show an NMDA-dependent short-term increase in synaptic strength (Ghiglieri et al, 2009). In the present experiments, intracellular recordings of FS interneurons recorded from corticostriatal slices of Bsn mutants showed that synaptic plasticity was completely blocked by a $20 \mathrm{~min}$ bath in vitro application of k252a $(200 \mathrm{nM})(p<0.01,5 \mathrm{~min}, p<0.05$, 10 min after the tetanus, $B s n$ mutant + k252a $v s B s n$ mutant) (Figure 2a). We also tested whether mutants receiving subchronic k252a and showing normal procedural learning performances also showed a reversal of short-term potentiation in FS interneurons. As expected, after in vivo TrkB antagonism, the plasticity was no longer expressed by FS interneurons of epileptic-treated mice $(p<0.001,5 \mathrm{~min}$, $p<0.01,10 \mathrm{~min}$ after the tetanus, $B s n$ mutant + vehicle $v s$ Bsn mutant $+\mathrm{k} 252 \mathrm{a}$ ) (Figure $2 \mathrm{~b}$ ).

\section{Synaptic Trkb Levels and PV/BDNF Colocalization are Increased in the Epileptic Striatum}

To measure the BDNF abundance in the striatum, with particular focus on FS interneurons, we performed an immunoblot quantification of TrkB levels and a double PV/BDNF and Calb/BDNF immunohistochemistry in the dorsolateral striatum. Levels of TrkB were quantified by western blot analysis of striatal samples obtained from

wild-type and $B s n$ mutant mice. Interestingly, while the TrkB expression in striatal homogenates obtained from mutant mice was similar to wild-types ( $97 \pm 6.8 \%$ of wild-type level), the amount of TrkB in the PSD-enriched fraction (TIF) of mutant striata was 1.5 -fold higher $(156 \pm 6.8 \%$ of wild-type level, $p<0.01$ ) (Figure 3). Increase in PSD association of TrkB suggests that changes in $\mathrm{BDNF} / \mathrm{TrkB}$ signaling pathway may also occur in the epileptic striatum.

Comparison of striatal PV/BDNF-positive cells quantification between the two genotypes showed a significantly higher PV/BDNF-colocalization in Bsn mutant mice than observed in wild-type littermates $(p<0.01)$ (Figures $4 \mathrm{a}$ and $\mathrm{b}$ ). Interestingly, the BDNF immunoreactivity was not changed in the MS neurons of epileptic mice, as striatal Calb/BDNF colocalization was similar to wild-types. These findings indicate that, in physiologic conditions, BDNF protein is found preferentially in association with MS neurons rather than with FS interneurons, while in the epileptic condition this trend is reversed (Figure $4 \mathrm{~b}$, see also Figure 5a). The increased BDNF localization in PV-positive interneurons in the mutant mice may account for the observed short-term plasticity in this neuronal subtype.

\section{Chronic Antiepileptic Treatment with VPA Rebalances TrkB Levels and Restores BDNF Distribution Between MS Neurons and FS Interneurons}

Chronic exposure to VPA, a broad-spectrum AED, has been reported to prevent the occurrence of short-term plasticity
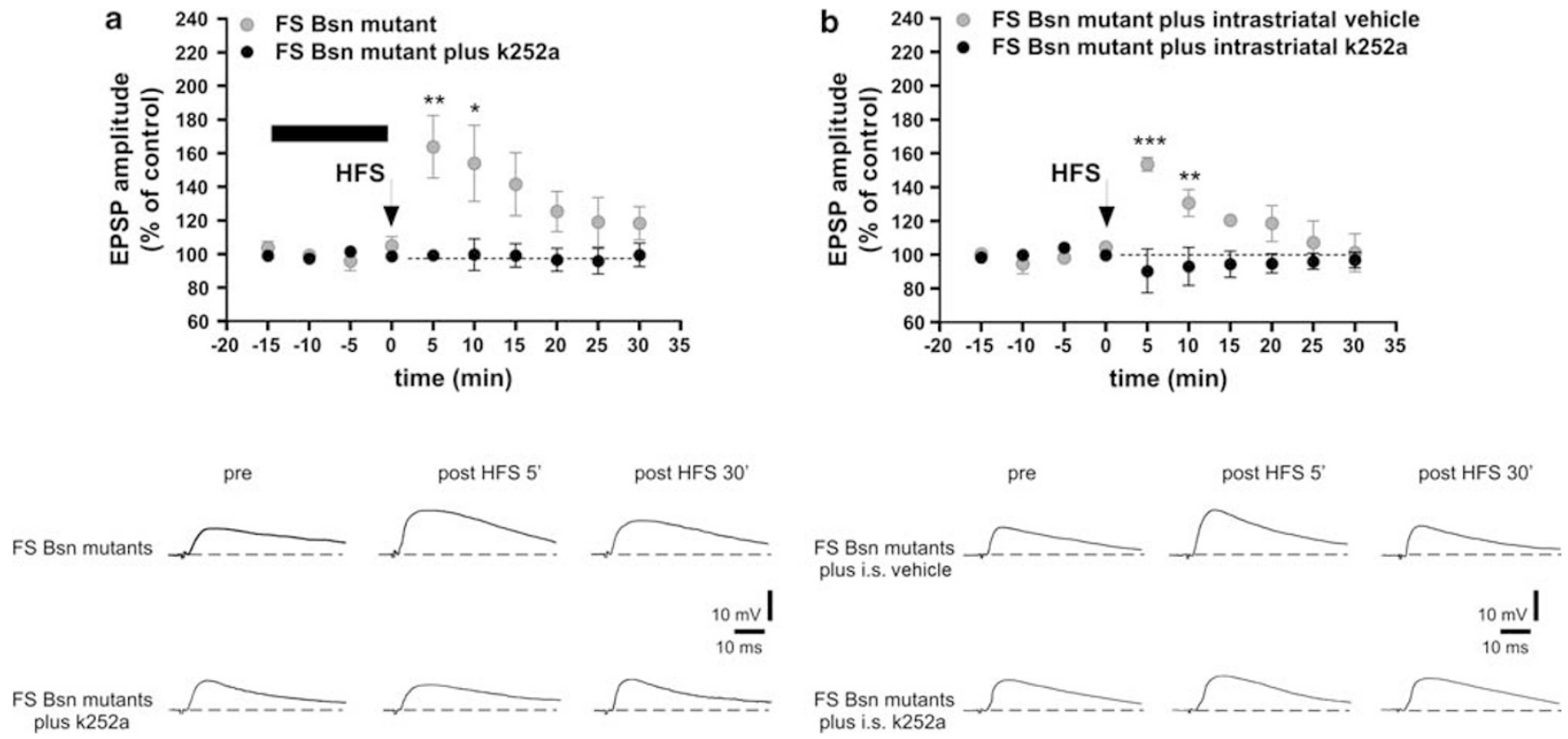

Figure 2 TrkB-dependent short-term plasticity in FS interneurons of Bsn mutant mice. (a) High-frequency stimulation (HFS, arrow) of corticostriatal pathway induced a synaptic potentiation that lasted 10-15 min in FS interneurons recorded intracellularly from corticostriatal slices of Bsn mutant mice. Conversely, 20-min bath application of the TrkB receptor antagonist k252a (200 nM), fully blocked the short-term potentiation (** $p<0.01,5$ min, *p $<0.05$, 10 min after the tetanus, Bsn mutant + k252a vs Bsn mutant, $n=6$ ). Bottom panels show example traces of excitatory postsynaptic potential (EPSP) from FS interneuron recorded in Bsn mutants corticostriatal slices (upper traces) and Bsn mutant slices with application of k252a (lower traces). (b) EPSP amplitudes of FS interneurons recorded ex vivo from corticostriatal slices of mice that received intrastriatal administration of k252a $(20 \mu \mathrm{M})$ or vehicle are shown in the upper panel. Similarly to what observed in naïve mutants, HFS was able to induce a short-term potentiation in mutants injected with vehicle but not in epileptic mice that received intrastriatal $\mathrm{k} 252 \mathrm{a}$ ( $* * * * 0<0.001,5 \mathrm{~min}$, $* * * 0.01,10 \mathrm{~min}$ after the tetanus, Bsn mutant + vehicle vs Bsn mutant $+k 252 \mathrm{a}, n=5$ ). Traces on the bottom represent examples of EPSP of FS interneurons recorded corticostriatal slices from vehicle-injected Bsn mutants (upper traces) and Bsn mutant that received intrastriatal administration of $k 252 a$ (lower traces). 

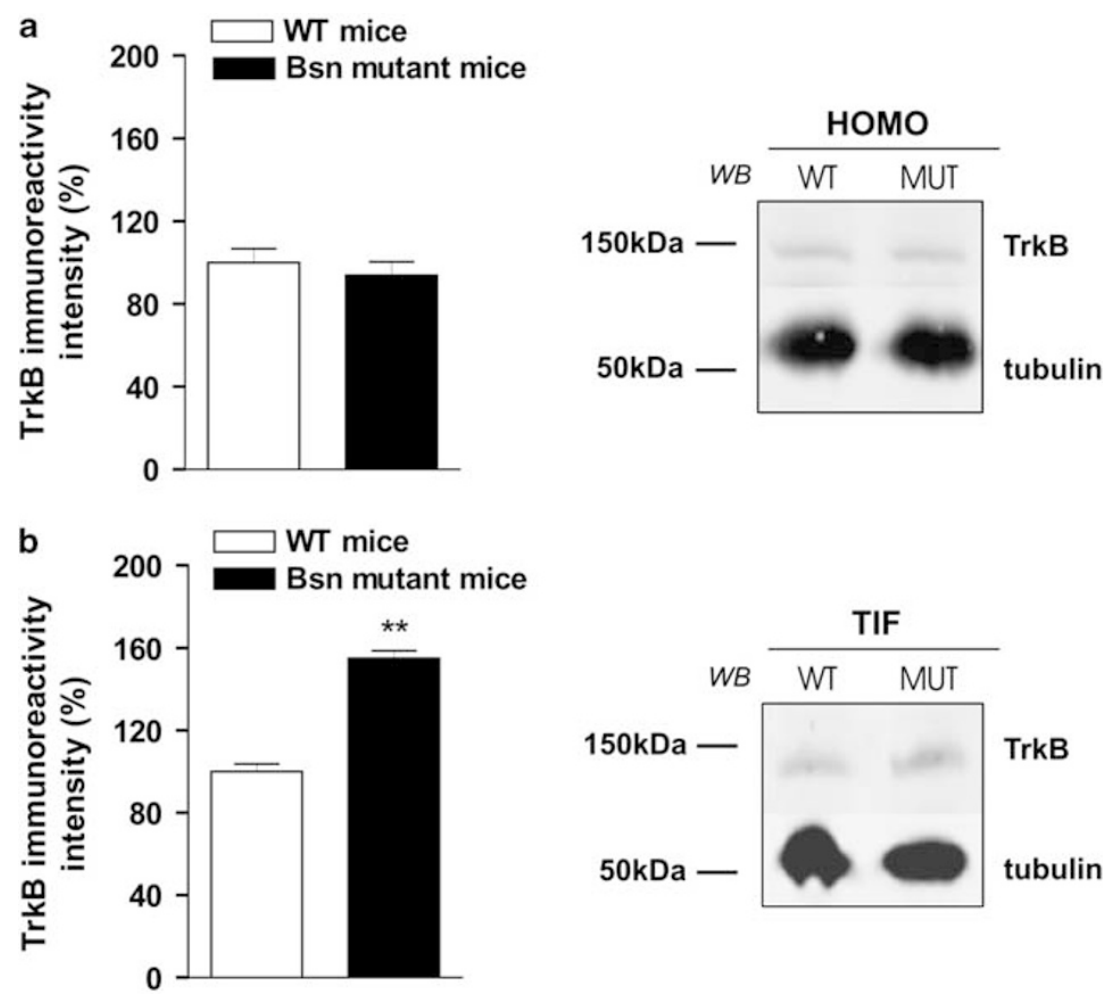

Figure 3 Striatal TrkB levels in Bsn mutant mice. Histogram shows the quantification of results of western blotting (WB) performed in homogenates (HOMO) (panel a) and Triton-insoluble fractions (TIF) (panel b) ( $p>0.05$, HOMO; *** $p<0.0$ I TIF). Representative immunoblots of striatal homogenates and TIFs from striata of wild-type (WT) and Bsn mutant mice are shown on the right of panels (a) and (b), respectively. The same amount of protein was loaded per lane, and tubulin was used as loading control in both homogenates and TIF fractions.

a

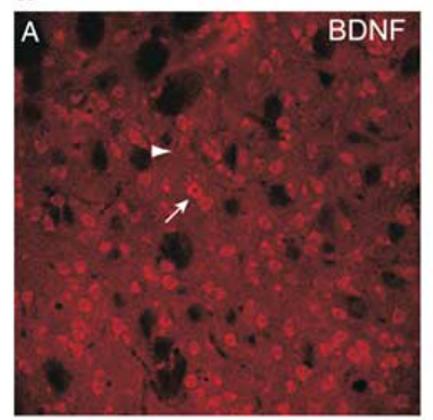

D

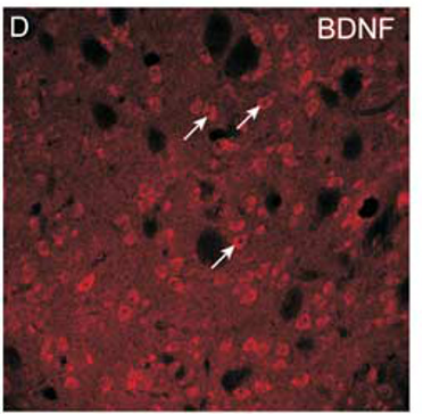

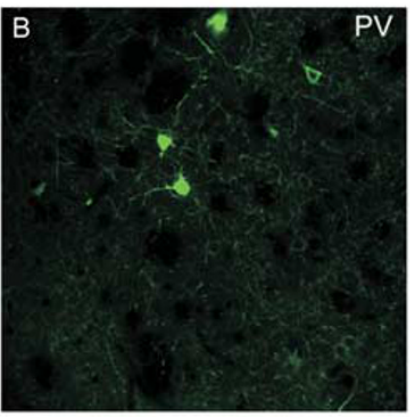

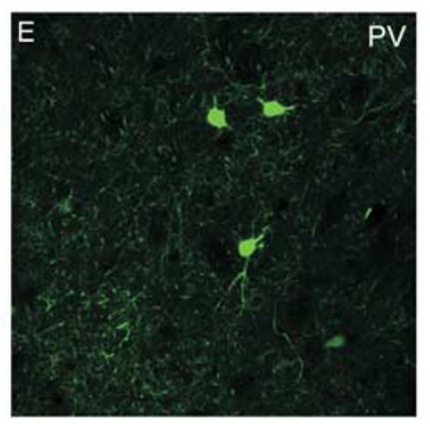

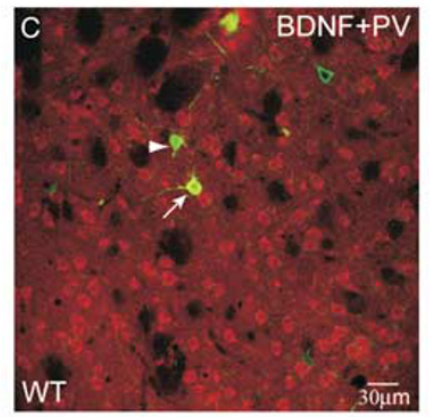

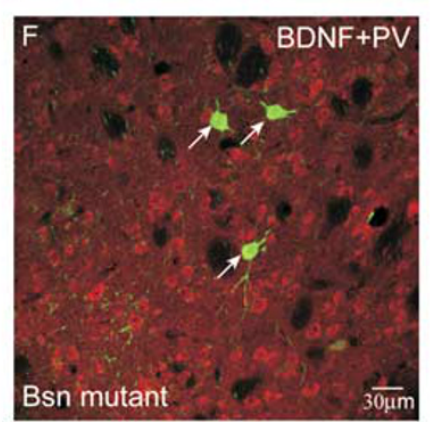

b BDNF immunoreactivity in Bsn mutant striatum

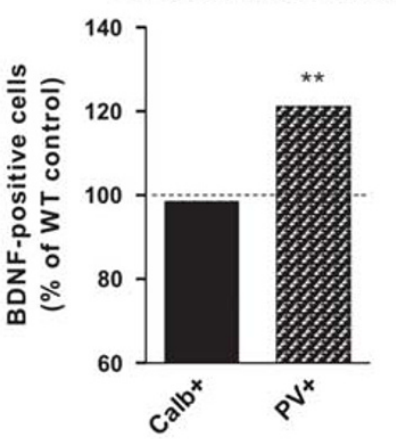

Figure 4 Striatal BDNF distribution in epileptic mice. (a) Comparison of striatal PV/BDNF and Calb/BDNF-positive cells quantification between the two genotypes showed a higher number of FS interneurons expressing BDNF protein in Bsn mutants than observed in wild-type controls (** $2<0.0 \mathrm{I}$ ). (b) Confocal laser scanning microscopy (CLSM) images show double-labeled immunofluorescence for BDNF and PV in wild-type (A-B-C) and Bsn mutant $(\mathrm{D}-\mathrm{E}-\mathrm{F})$ mice. BDNF immunolabeling is visualized in red-cy3 fluorescence and PV is visualized in green-cy2 fluorescence. The merged image is shown as a yellow fluorescence, which coincides with the colocalization between BDNF and PV. The arrows show the colocalization of PV-positive interneurons with BDNF. The arrowheads show the no BDNF-containing PV interneurons. 
a

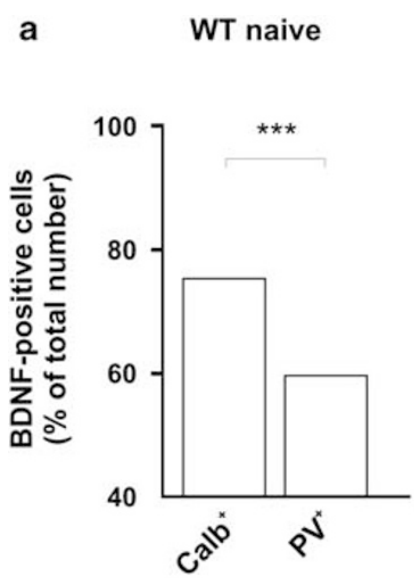

Bsn mutant naive
WT plus VPA

plus VPA

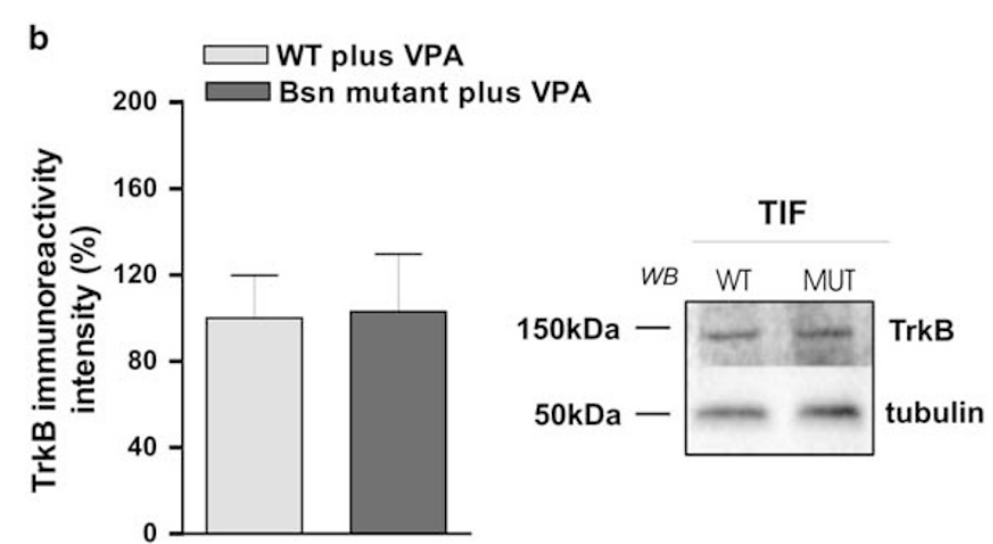

Figure 5 VPA effects on striatal BDNF distribution pattern. (a) The histograms show the percent of BDNF-positive FS interneurons and MS neurons in the striatum of naïve and VPA-treated wild types and Bsn mutants. Although in wild-type mice, the proportion of MS Calb-positive neurons that express BDNF protein was higher than the relative number of PV/BDNF-positive interneurons (**** $p<0.00 \mathrm{I}$ ), in epileptic mice the immunoreactivity of BDNF was similar in both neuronal subtypes. Chronic early treatment with VPA restored the ratio between PV/BDNF and Calb/BDNF cells in the striatum of epileptic mice $(* * * * 0.001)$ to control levels. Early exposure to VPA in wild types increased the neurotrophin immunoreactivity in both neuronal subtypes, preserving the ratio between BDNF-positive MS neurons and FS interneurons that was similar to the one observed in naive controls (**** $p<0.00 \mathrm{I}$ ). (b) After chronic treatment with VPA synaptic levels of striatal TrkB were similar in both Bsn mutant and wild-type mice $(p>0.05)$. Histograms show the quantification of western blotting performed in TIF fractions.

in FS interneurons of $B s n$ mice by reducing the frequency of the epileptic seizures (Ghiglieri et al, 2009). To clarify whether changes of BDNF distribution may depend on chronic seizure activity, we analyzed the expression of BDNF protein into PV-positive (FS interneurons) and calbpositive (MS neurons) cells by means of double-label immunofluorescence in mice receiving chronic antiepileptic treatment. VPA $(400 \mathrm{mg} / \mathrm{kg} /$ die $)$ was given orally to heterozygous females, to treat pups by maternal breast milk, from postnatal day $(\mathrm{P}) 0$ to weaning $(\mathrm{P} 21)$. Treatment was then extended into adulthood until the day of killing of wild-type and mutant mice.

Our results show that while the mutant condition was associated with a similar BDNF immunoreactivity in FS interneurons and MS neurons, because of increased colocalization with PV, early treatment of epileptic mice with chronic VPA was able to restore the ratio of BDNF distribution between the two neuronal subtypes $(p<0.001)$ (Figure $5 \mathrm{a}$, last graph on the right). In wild-type mice, although VPA exerted an increased protein expression in both PV and Calb, the pattern of BDNF distribution was preserved as the number of MS neurons found positive to BDNF was significantly higher than the number of
PV-positive interneurons colocalizing with the neurotrophin $(p<0.001)$ (Figure 5a, third graph). In line with this, after early chronic VPA treatment striatal synaptic levels of TrkB were similar in the two genotypes (Figure 5b).

\section{DISCUSSION}

The main finding of this study is that changes of TrkB/ BDNF system underlie striatal behavioral and plastic alterations observed in epileptic mice lacking the presynaptic protein Bassoon.

In $B s n$ mutants, striatal neuronal subtypes are differently sensitive to recurrent paroxysmal activity (Ghiglieri et al, 2009). However, the behavioral correlates of neuronal abnormalities in this model of epilepsy have not been further characterized. In this study, we have shown that such reorganization of plasticity between MS neurons and FS interneurons was associated with an abnormal enhancement of procedural learning in epileptic mice. The first issue that arises from these data concerns the behavioral correlate of striatal plasticity. In Bsn mice, in fact, frequent cortical seizures alter synaptic plasticity of striatal MS 
neurons and NMDA receptor subunit composition while striatal FS interneurons are more abundant and express an adaptive form of NMDA-dependent plasticity (Ghiglieri et al, 2009). In this scenario, given that MS projecting neurons constitute the large majority of neuronal cells in the striatum, one would expect to observe poor procedural learning performances in mutants rather than increase in conditioned responses. One possible explanation of this discrepancy might be that the enhanced feedforward inhibition, because of FS interneuronal plasticity, may somehow result in an improvement of striatal functions. This advantage may compensate the hippocampal deficit that characterizes this model. In fact, it has been shown that Bsn mutants show impaired synaptic functions in the hippocampus, that is, lower excitability, probably because of a reduced number of active excitatory synapses (Altrock et al, 2003). This is associated with a decrease in LTP and an impaired acquisition of hippocampus-dependent non-spatial memory (Sgobio et al, 2010). Under these conditions, it is possible that competitive roles of corticostriatal and hippocampal systems in learning behaviors may occur according to converging evidences from experimental and clinical studies (Gabrieli, 1995; McDonald and White, 1993; Packard and McGaugh, 1996; Packard and White, 1991). In particular, in animals in which hippocampal functions are compromised, procedural learning, which relies on striatal memory system, is improved and a competition of the two hippocampal and striatal memory systems may become evident when activated simultaneously to provide a correct learned solution in a specific task (Poldrack and Packard, 2003). Thus, the enhancement of procedural learning performances in this epileptic model can be interpreted as a result of the imbalance between striatal and hippocampal memory systems. The first interesting aspect of this study is that changes in BDNF/ TrkB system seem to underlie neuronal modifications leading to altered behavioral response. In fact, in vivo intracerebral subchronic administration of $\operatorname{TrkB}$ receptor inhibitor was able to reset striatal-dependent learning to control conditions. Interestingly, ex vivo intracellular recordings, showed that abnormal plasticity of FS interneurons was absent in corticostriatal slices obtained from $B s n$ mutants that received intrastriatal $\mathrm{k} 252 \mathrm{a}$. Consistent with these results, in vitro acute application of $\mathrm{k} 252 \mathrm{a}$ on corticostriatal slices obtained from epileptic mice was sufficient to inhibit the induction of pathologic short-term plasticity in FS GABAergic interneurons. Along this line, if plasticity of FS interneurons relies on $\operatorname{TrkB}$ receptor activation one would expect that in epileptic condition BDNF levels may also be changed in this striatal population. To clarify this point, we performed an immunohistochemical study of BDNF distribution in the dorsolateral striatum of epileptic mice. Our results revealed that while in control mice the neurotrophin is more abundant in MS neurons than in FS interneurons, in epileptic condition the relative amount of FS PV-positive interneurons colocalizing with BDNF was significantly higher than observed in wild-type mice, suggesting that a specificity of action of BDNF on FS interneurons may subsist.

Consistent with the idea that striatal neuronal subtypes are differently sensitive to recurrent paroxysmal activity, these data seem to suggest that BDNF preferentially interacts with TrkB receptors expressed on FS interneurons. Cell-type specificity is a common feature for neurotrophins, since effectiveness of BDNF is critically influenced by neuronal activity and synaptic potentiation induced by BDNF can be selective for active synapses. Accordingly, BDNF exerts opposite effects on cortical pyramidal neurons and GABAergic interneurons, depending on the level of neuronal activity (Rutherford et al, 1998). Moreover, several studies pointed out to the specific role of BDNF in the differentiation of GABAergic terminals (Bolton et al, 2000) and in particular on the development and maturation of GABAergic PV-positive interneurons (Berghuis et al, 2004). In this study, we have provided the first evidence that synaptic levels of $\operatorname{TrkB}$ are higher in the striatum of epileptic mice supporting the idea that also BDNF levels may increase during epilepsy as observed for other brain areas subjected to chronic seizure activity. In the striatum, low concentrations of BDNF, through its high-affinity receptor TrkB, activate different calcium-dependent intracellular pathways in a very precise mode, resulting in accurate tuning of MS neuronal activity. It is then possible that in these epileptic mice excessive discharge activity during early cortical seizures may induce increases of BDNF levels. Consequently, an alternative pattern of BDNF distribution may be established to favor an enhancement of inhibitory control within the striatum as adaptive mechanism. On this view, the increased presence of the neurotrophin at interneuronal level may account for the emergence of plasticity in FS interneurons with the concomitant reduction in LTP observed in the projecting neurons (Ghiglieri et al, 2009). Such adaptive response may protect MS neurons from excessive activity and preserve striatal functions. Our results are in line with a recent report showing that BDNF preferentially stimulates active synapses (Nagappan and Lu, 2005). In fact, in epileptic mice, while MS neurons show reduced LTP, FS interneurons, which are normally able to sustain higher neuronal activities, show a form of synaptic plasticity that critically requires the activation of NMDA receptor (Ghiglieri et al, 2009). It is then possible that this particular feature makes FS interneurons a preferential target for the neurotrophin. Early treatment of Bsn mutants with chronic VPA, by reducing seizures, rescues LTP in MS neurons and prevents the induction of adaptive plasticity in FS interneurons (Ghiglieri et al, 2009). However, the mechanism by which the reduction of seizures may induce a rebalance of synaptic plasticity has been difficult to explore. In this study, we tested the hypothesis that early exposure to AED might rebalance TrkB levels and BDNF distribution to normalize the physiological synaptic plasticity within the striatal MS neurons and FS interneurons. Our results show that, after chronic antiepileptic treatment, mutant mice showed a more physiological distribution of striatal BDNF protein between MS neurons and FS interneurons, similar to the one observed in wild-type controls. These data suggest that, by decreasing the frequency of seizures, VPA may also restore striatal synaptic TrkB and BDNF to control levels bringing to a physiological action of BDNF. The reduced effect of BDNF on FS interneurons may then clarify the restorative effect of chronic VPA on striatal synaptic plasticity (Ghiglieri et al, 2009). This study provides a first indication that BDNF is involved in the development of 
striatal alterations underlying in the early-onset epileptic syndrome associated with the absence of presynaptic protein Bassoon. In vivo direct or indirect interferences on TrkB/BDNF system by either inhibition of TrkB tyrosine kinases or chronic VPA treatment showed that behavioral alterations of mutants are paralleled by higher synaptic TrkB levels, emergence of interneuronal plasticity and increased BDNF immunoreactivity at interneuronal level. These evidences clearly suggest that in epileptic mice profound alterations of TrkB/BDNF levels occur, leading to a pathological redistribution of BDNF between the two neuronal subtypes. Although such adaptive response may protect MS neurons from excessive activity and originally preserve striatal functions, alterations of TrkB/BDNF system may lead to pathological actions of BDNF underlying recurrence of seizures. Early treatment with VPA, able to limit the seizures, prevents this cell-type-specific alteration and restores striatal plasticity to control levels. These effects argue for a strong association between TrkB/BDNF changes and altered corticostriatal plasticity. At present it is, however, unclear whether $B s n$ deficiency directly affects BDNF secretion. Moreover, given the complex role of growth factors in the induction and maintenance of longlasting adaptive changes, the question whether BDNF alterations are causative of epilepsy rather than being a result of recurring seizures is far from being resolved. It is worth mentioning that because of its involvement in activity-dependent synaptic plasticity BDNF has been recently suggested as a new therapeutic target in the treatment of other neurological disorders, such as neurodegenerative diseases (Zuccato and Cattaneo, 2009), drug abuse (Russo et al, 2009), and depression (Castren et al, 2007). Interestingly, all these pathologies share the common feature of maladaptive learning. In particular, recent evidences have suggested that abnormal regional-specific BDNF expression contributes to the functional defects observed in drug addiction (Fumagalli et al, 2007) and in depression (Molteni et al, 2009). In this scenario, our data shed new light on the complex role of BDNF in epilepsy and within the striatal microcircuit. However, additional studies are required to unravel these interesting effects of BDNF regulation on striatal activity.

\section{ACKNOWLEDGEMENTS}

This work was supported by the European Community Contract Number 222918 (REPLACES) FP7-Thematic priority HEALTH (PC, EG), Ministero della Salute Progetto Strategico 2007 (PC, BP), and Progetti Finalizzati e Ricerca Corrente IRCCS Ministero della Salute 2006-2008 (PC, BP).

\section{DISCLOSURE}

The authors declare no conflict of interest.

\section{REFERENCES}

Altrock WD, tom Dieck S, Sokolov M, Meyer AC, Sigler A, Brakebusch C et al (2003). Functional inactivation of a fraction of excitatory synapses in mice deficient for the active zone protein bassoon. Neuron 37: 787-800.
Bartrup JT, Moorman JM, Newberry NR (1997). BDNF enhances neuronal growth and synaptic activity in hippocampal cell cultures. Neuroreport 8: 3791-3794.

Berghuis P, Dobszay MB, Sousa KM, Schulte G, Mager PP, Hartig $\mathrm{W}$ et al (2004). Brain-derived neurotrophic factor controls functional differentiation and microcircuit formation of selectively isolated fast-spiking GABAergic interneurons. Eur J Neurosci 20: 1290-1306.

Bolton MM, Pittman AJ, Lo DC (2000). Brain-derived neurotrophic factor differentially regulates excitatory and inhibitory synaptic transmission in hippocampal cultures. J Neurosci 20: 3221-3232.

Calabresi P, Pisani A, Mercuri NB, Bernardi G (1992). Long-term potentiation in the striatum is unmasked by removing the voltage-dependent magnesium block of NMDA receptor channels. Eur J Neurosci 4: 929-935.

Castren E, Voikar V, Rantamaki T (2007). Role of neurotrophic factors in depression. Curr Opin Pharmacol 7: 18-21.

Fumagalli F, Di Pasquale L, Caffino L, Racagni G, Riva MA (2007). Repeated exposure to cocaine differently modulates BDNF mRNA and protein levels in rat striatum and prefrontal cortex. Eur J Neurosci 26: 2756-2763.

Gabrieli JD (1995). A systematic view of human memory processes. J Int Neuropsychol Soc 1: 115-118.

Gall CM (1993). Seizure-induced changes in neurotrophin expression: implications for epilepsy. Exp Neurol 124: 150-166.

Ghiglieri V, Picconi B, Sgobio C, Bagetta V, Barone I, Paille V et al (2009). Epilepsy-induced abnormal striatal plasticity in Bassoon mutant mice. Eur J Neurosci 29: 1979-1993.

Humpel C, Wetmore C, Olson L (1993). Regulation of brainderived neurotrophic factor messenger RNA and protein at the cellular level in pentylenetetrazol-induced epileptic seizures. Neuroscience 53: 909-918.

Isackson PJ, Huntsman MM, Murray KD, Gall CM (1991). BDNF mRNA expression is increased in adult rat forebrain after limbic seizures: temporal patterns of induction distinct from NGF. Neuron 6: 937-948.

Jankowsky JL, Patterson PH (2001). The role of cytokines and growth factors in seizures and their sequelae. Prog Neurobiol 63: $125-149$.

Lu B (2003). BDNF and activity-dependent synaptic modulation. Learn Mem 10: 86-98.

Marty S, Berninger B, Carroll P, Thoenen H (1996). GABAergic stimulation regulates the phenotype of hippocampal interneurons through the regulation of brain-derived neurotrophic factor. Neuron 16: 565-570.

McDonald RJ, White NM (1993). A triple dissociation of memory systems: hippocampus, amygdala, and dorsal striatum. Behav Neurosci 107: 3-22.

Merlio JP, Ernfors P, Kokaia Z, Middlemas DS, Bengzon J, Kokaia M et al (1993). Increased production of the TrkB protein tyrosine kinase receptor after brain insults. Neuron 10: 151-164.

Molteni R, Cattaneo A, Calabrese F, Macchi F, Olivier JD, Racagni G et al (2009). Reduced function of the serotonin transporter is associated with decreased expression of BDNF in rodents as well as in humans. Neurobiol Dis, print copy in press (originally published online 23 December 2009, at http://dx.doi.org/10.1016/ j.nbd.2009.12.014).

Nagappan G, Lu B (2005). Activity-dependent modulation of the BDNF receptor TrkB: mechanisms and implications. Trends Neurosci 28: 464-471.

Packard MG, McGaugh JL (1996). Inactivation of hippocampus or caudate nucleus with lidocaine differentially affects expression of place and response learning. Neurobiol Learn Mem 65: 65-72.

Packard MG, White NM (1991). Dissociation of hippocampus and caudate nucleus memory systems by posttraining intracerebral injection of dopamine agonists. Behav Neurosci 105: 295-306.

Paxinos G, Franklin KBJ (2007). The Mouse Brain in Stereotaxic Coordinates Second Edition. Academic Press: San Diego. 
Poldrack RA, Packard MG (2003). Competition among multiple memory systems: converging evidence from animal and human brain studies. Neuropsychologia 41: 245-251.

Poo MM (2001). Neurotrophins as synaptic modulators. Nat Rev Neurosci 2: 24-32.

Russo SJ, Mazel-Robinson MS, Ables JL, Nestler EJ (2009). Neurotrophic factors and structural plasticity in addiction. Neuropharmacology 56(Suppl 1): 73-82.

Rutherford LC, Nelson SB, Turrigiano GG (1998). BDNF has opposite effects on the quantal amplitude of pyramidal neuron and interneuron excitatory synapses. Neuron 21: 521-530.

Salin T, Mudo G, Jiang XH, Timmusk T, Metsis M, Belluardo N (1995). Up-regulation of TrkB mRNA expression in the rat striatum after seizures. Neurosci Lett 194: 181-184.

Schmidt-Kastner R, Humpel C, Wetmore C, Olson L (1996). Cellular hybridization for BDNF, TrkB, and NGF mRNAs and BDNF-immunoreactivity in rat forebrain after pilocarpineinduced status epilepticus. Exp Brain Res 107: 331-347.

Sgobio C, Ghiglieri V, Costa C, Bagetta V, Siliquini S, Barone I et al (2010). Hippocampal synaptic plasticity, memory, and epilepsy: effects of long-term valproic acid treatment. Biol Psychiatry, print copy in press (originally published online 13 January 2010, at http://journals.elsevierhealth.com/periodicals/bps/inpress).

Takahashi M, Hayashi S, Kakita A, Wakabayashi K, Fukuda M, Kameyama S et al (1999). Patients with temporal lobe epilepsy show an increase in brain-derived neurotrophic factor protein and its correlation with neuropeptide Y. Brain Res 818: 579-582.

White NM, McDonald RJ (2002). Multiple parallel memory systems in the brain of the rat. Neurobiol Learn Mem 77: 125-184.

Wyneken U, Marengo JJ, Villanueva S, Soto D, Sandoval R, Gundelfinger ED et al (2003). Epilepsy-induced changes in signaling systems of human and rat postsynaptic densities. Epilepsia 44: 243-246.

Wyneken U, Smalla KH, Marengo JJ, Soto D, de la Cerda A, Tischmeyer $\mathrm{W}$ et al (2001). Kainate-induced seizures alter protein composition and N-methyl-D-aspartate receptor function of rat forebrain postsynaptic densities. Neuroscience 102: 65-74.

Yamada MK, Nakanishi K, Ohba S, Nakamura T, Ikegaya Y, Nishiyama $\mathrm{N}$ et al (2002). Brain-derived neurotrophic factor promotes the maturation of GABAergic mechanisms in cultured hippocampal neurons. J Neurosci 22: 7580-7585.

Zuccato C, Cattaneo E (2009). Brain-derived neurotrophic factor in neurodegenerative diseases. Nat Rev Neurol 5: 311-322.

Supplementary Information accompanies the paper on the Neuropsychopharmacology website (http://www.nature.com/npp) 